\title{
Modulation by Central and Basolateral Amygdalar Nuclei of Dopaminergic Correlates of Feeding to Satiety in the Rat Nucleus Accumbens and Medial Prefrontal Cortex
}

\author{
Soyon Ahn and Anthony G. Phillips \\ Department of Psychiatry and the Brain Research Centre, University of British Columbia, Vancouver, Canada V6T 2A1
}

Current studies raise the possibility that subregions within the amygdala may interact with the mesocorticolimbic dopamine (DA) system to subserve specific psychological processes underlying food reward. The present study compared the effect of reversible inactivation of the central nucleus $(\mathrm{CeN})$ versus the basolateral amygdala (BLA) on DA efflux in the nucleus accumbens (NAC) and medial prefrontal cortex (mPFC) in hungry rats that were tested in a food-devaluation procedure. During DA microdialysis experiments, lidocaine, a sodium channel blocker, was delivered via reverse dialysis into the CeN or BLA while rats were given two consecutive meals of Froot Loops. Loss of CeN function impaired the development of satiety during an initial meal and, consequently, diminished the effect of devaluation by satiety on intake of the same food during a second meal. Inactivation of

Two dissociable psychological processes are proposed to underlie food reward: an evaluation of the hedonic properties of food and the motivation to eat (Berridge and Robinson, 1998). Hedonic responses believed to reflect the "liking" of food depend on benzodiazepine circuits in the brainstem (Berridge and Treit, 1986; Berridge, 1996), as well as GABAergic, glutamatergic, and opioid circuits in the forebrain (Bechara and van der Kooy, 1992; Bakshi and Kelley, 1994; Maldonado-Irizarry et al., 1995; Stratford and Kelley, 1997). Motivation related to "wanting" involves activation of the mesocorticolimbic dopamine (DA) pathway (Mogenson and Phillips, 1976; Berridge, 1996). Specifically, DA activity has been hypothesized to mediate the attribution of incentive salience to environmental stimuli associated with a primary reward (Robinson and Berridge, 1993), a process that may allow conditioned stimuli to serve as incentives themselves or guide an animal into contact with a primary reward.

Important questions remain regarding the neural mechanisms by which changes in motivation, such as those that accompany satiety, can influence the reward value of food. A key role in this process may be played by the amygdala, a forebrain structure that receives sensory information (e.g., olfactory and gustatory) from the brainstem and the cortex, as well as physiological signals related to hunger and satiety via brainstem nuclei (Mei, 1994; Zeigler, 1994; Norgren, 1995; Shipley et al., 1995; Woods et al.,

Received July 22, 2002; revised Sept. 3, 2002; accepted Oct. 1, 2002.

This work was funded by a research grant from the Canadian Institutes for Health Research (A.G.P.). We gratefully acknowledge the assistance of Dr. Natalia Gorlova for advice on the procedure for reverse dialysis of lidocaine and Kitty So for her meticulous work in preparing the dialysis probes.

Correspondence should be addressed to Dr. A. G. Phillips, Department of Psychiatry, University of British Columbia, 2255 Wesbrook Mall, Vancouver, British Columbia, Canada V6T 2A1. E-mail: aphillips@cortex.psych.ubc.ca.

Copyright (C) 2002 Society for Neuroscience 0270-6474/02/2210958-08\$15.00/0 the $\mathrm{CeN}$ was also associated with decreased basal levels of DA efflux in the NAc before food intake and attenuated increases in DA efflux related to anticipatory and consummatory aspects of feeding in both the NAc and mPFC. In contrast, inactivation of the BLA did not affect feeding behavior or DA efflux. Overall, these findings indicate that the $\mathrm{CeN}$ and BLA independently modulate DA transmission in both terminal regions. It is proposed that interaction between the $\mathrm{CeN}$ and mesocorticolimbic DA activity may be a mechanism by which hunger and satiety signals influence the value of food reward, or alternatively, a mechanism by which memory for a recently consumed food regulates food intake.

Key words: microdialysis; reverse dialysis; lidocaine; reversible inactivation; incentive devaluation; food reward

1998). In previous studies, impairments in food-related behaviors were reported in monkeys and rats with amygdala lesions, including indiscriminate sampling of nonfood items, altered food preferences, and interference with reinforcer devaluation effects (Box and Mogenson, 1975; Aggleton and Passingham, 1982; Murray et al., 1996; Màlkovà et al., 1997). These observations, together with known neuroanatomical connections, suggest that the amygdala may serve to interface external and internal sensory information with the motivational systems of the brain (Rolls, 1999), namely the mesocorticolimbic DA system. More recent studies of functional amygdalar circuitry have focused on two subregions delineated by anatomical connectivity and immunohistochemical markers, the central nucleus $(\mathrm{CeN})$ and the basolateral amygdala (BLA) (McDonald, 1991; Pitkänen et al., 1997; Swanson and Petrovich, 1998). The significance of each subregion in the rat has been examined with several behavioral learning paradigms, in both appetitively and aversively motivated contexts (Killcross et al., 1997; Everitt et al., 1999; Holland and Gallagher, 1999). Of particular relevance to food reward are reports that the $\mathrm{CeN}$ and the BLA are involved in the coding of incentive value of food (Kesner et al., 1989; Uwano et al., 1995; Salinas et al., 1996).

In view of the above considerations, an interaction between the $\mathrm{CeN}$ and/or the BLA with DA transmission in the nucleus accumbens (NAc) and/or the medial prefrontal cortex (mPFC) may be a mechanism by which internal motivational signals, such as hunger and satiety, determine the incentive value of food. In a recent study, we reported that the $\mathrm{CeN}$ and the BLA differentially influence basal and feeding-evoked changes in DA efflux in both terminal regions (Ahn and Phillips, 2002). To explore further the relevance of these interactions in feeding behavior, the present study compared the effect of reversible inactivation of the $\mathrm{CeN}$ versus the BLA on DA efflux in the NAc and $\mathrm{mPFC}$ in rats that 
were tested in a food-devaluation procedure. During microdialysis experiments, hungry rats were reverse dialyzed with lidocaine, a sodium channel blocker, into the $\mathrm{CeN}$ or the BLA before and during an initial meal of Froot Loops. Forty minutes later, rats were presented with a second meal of Froot Loops to investigate possible post-lidocaine effects on intake of food devalued by satiety. A previous finding that inactivation of the BLA triggers oscillatory changes in DA efflux in the mPFC precluded the assessment of feeding-evoked changes in DA levels in this terminal region (Ahn and Phillips, 2002).

\section{MATERIALS AND METHODS}

All experimental protocols were approved by the Committee on Animal Care (University of British Columbia) and were conducted in compliance with guidelines provided by the Canadian Council of Animal Care.

\section{Subjects and surgery}

Twenty-six Long-Evans male rats (Charles River Canada, St. Constant, Quebec, Canada) were housed in a colony room maintained at $\sim 21^{\circ} \mathrm{C}$ with a $12 \mathrm{hr}$ light/dark cycle (lights on at 6:00 A.M.). Rats weighting $300 \pm 20$ gm were assigned randomly to one of three groups based on the structures of interest (CeN-NAc, CeN-mPFC, and BLA-NAc) and implanted with guide cannulas under xylazine $(7 \mathrm{mg} / \mathrm{kg}$, i.p.) and ketamine hydrochloride $(100 \mathrm{mg} / \mathrm{kg}$, i.p.) anesthesia. Stereotaxic coordinates for the CeN, BLA, NAc, and mPFC were based on Paxinos and Watson (1997). Nitric acid-passivated stainless steel guide cannulas (19 gauge, $15 \mathrm{~mm}$ ) for reverse dialysis were implanted bilaterally $1 \mathrm{~mm}$ below dura, directly above either the $\mathrm{CeN}[-2.3 \mathrm{~mm}$ anteroposterior (AP) and $\pm 4.0 \mathrm{~mm}$ mediolateral (ML) from bregma, $-7.7 \mathrm{~mm}$ dorsoventral (DV) from dura] or BLA ( $-3.0 \mathrm{~mm}$ AP and $\pm 5.0 \mathrm{~mm}$ ML from bregma, -8.4 $\mathrm{mm}$ DV from dura). Similarly, guide cannulas for microdialysis probes were implanted above the NAc $(+1.7 \mathrm{~mm} \mathrm{AP}$ and $\pm 1.1 \mathrm{~mm}$ ML from bregma, $-8.0 \mathrm{~mm} \mathrm{DV}$ from dura) or the $\mathrm{mPFC}(+3.0 \mathrm{~mm} \mathrm{AP}$ and \pm 0.6 $\mathrm{mm}$ ML from bregma, $-4.5 \mathrm{~mm}$ DV from dura).

After surgery, rats were housed individually in plastic bins with corncob bedding. After a 1 week period of recovery, rats were placed on a restricted feeding schedule (20-25 gm daily; Rat Diet 5012; PMI Feeds, Delta, British Columbia, Canada) to maintain their body weight at $85-90 \%$ of free-feeding weight. Water was available ad libitum. Novel objects (e.g., sterilized egg and milk cartons) were introduced into home bins weekly to promote exploratory and play behavior.

\section{Microdialysis and HPLC}

Microdialysis probes were concentric in design with silica inlet-outlet lines. The active surface consisted of a semipermeable membrane $2 \mathrm{~mm}$ in length (340 $\mu \mathrm{m}$ outer diameter; 65,000 molecular weight cutoff; Filtral 12; Hospal, Neurnberg, Germany). Probes were flushed continuously at $1 \mu \mathrm{l} / \mathrm{min}$ with a modified Ringer's solution [i.e., "perfusate" (in mM): 10 sodium phosphate, $1.2 \mathrm{CaCl}_{2}, 3.0 \mathrm{KCl}, 1.0 \mathrm{MgCl}_{2}$, and $147.0 \mathrm{NaCl}, \mathrm{pH}$ 7.4] using a $2.5 \mathrm{ml}$ gastight syringe (Hamilton, Reno, NV) and a syringe pump (model 22; Harvard Apparatus, South Natick, MA). Typical in vitro probe recoveries of DA conducted at room temperature were $18 \pm$ $1 \%$ of a standard DA solution. DA was separated from other chemical species present in microdialysis samples by HPLC and quantified by electrochemical detection. The details of the HPLC methods have been described previously (Fiorino et al., 1997; Ahn and Phillips, 1999).

\section{Reverse dialysis of lidocaine}

Probes used for reverse dialysis of lidocaine were a modified version of the microdialysis probes described above. The length of the active membrane surface was adjusted to cover the maximal dorsoventral extent of each structure: $1.2 \mathrm{~mm}$ for the $\mathrm{CeN}$ and $1.8 \mathrm{~mm}$ for the BLA (Paxinos and Watson, 1997). Reverse dialysis of lidocaine involved replacing normal perfusate that flowed through the probe with perfusate containing $2 \%$ lidocaine hydrochloride $(20 \mathrm{mg} / \mathrm{ml}$; Research Biochemicals, Natick, MA) for a 70 min interval. The rate of flow through the probe was maintained at $1 \mu \mathrm{l} / \mathrm{min}$ at all times.

\section{Apparatus and protocol for feeding paradigm}

All habituation sessions and experiments were conducted from 8:00 A.M. to $2:: 00$ P.M. in a Plexiglas chamber $(42 \times 38 \times 38 \mathrm{~cm})$ fitted with a perforated screen and a two-channel liquid swivel (Instech Laboratories,
Plymouth Meeting, PA). Two weeks into the restricted feeding schedule described above, rats were habituated twice (days 1 and 2) to being tethered to the liquid swivel by a stainless steel coil and also presented with the test food Froot Loops, a fruit-flavored cereal (Kellogg Canada, London, Ontario, Canada), to overcome neophobia. On day 3, rats were given a trial run of the two-meal feeding paradigm. At the start of each meal, $3 \mathrm{gm}$ of Froot Loops was presented in the food bin behind a screen for $10 \mathrm{~min}$ (the appetitive period), during which animals could see and smell the food. The screen was then removed, and animals had access to the food for $10 \mathrm{~min}$, after which the remaining food, if any, was replaced by another $3 \mathrm{gm}$ of food every $10 \mathrm{~min}$ for a total of $40 \mathrm{~min}$ (the consummatory period). After a 40 min recess, a second meal of Froot Loops was presented in the same manner as the first meal.

\section{Microdialysis experiments}

On days 5 and 6, microdialysis experiments were conducted during the two-meal feeding paradigm described above. Each animal was tested in two conditions in random order, one involving bilateral reverse dialysis of lidocaine in the $\mathrm{CeN}$ or BLA and the other continuous flushing of the probe with normal perfusate.

Fourteen to $16 \mathrm{hr}$ before the collection of the first sample, microdialysis probes were implanted unilaterally into either the NAc or mPFC, and reverse-dialysis probes were placed bilaterally into either the $\mathrm{CeN}$ or BLA. For the purpose of controlling for potential hemispheric differences, the left and right NAc or mPFC of each subject were randomly assigned to the lidocaine or control condition. Animals remained overnight in the test chamber, with their daily ration of food and ad libitum access to water. In the morning, dialysis samples were collected at $10 \mathrm{~min}$ intervals (i.e., $10 \mu \mathrm{l}$ ) from the NAc or mPFC and immediately assayed for DA using HPLC and electrochemical detection. Baseline sampling continued until four consecutive microdialysis samples showed $<5 \%$ fluctuation in DA content before beginning reverse dialysis of lidocaine or vehicle (normal perfusate) into the $\mathrm{CeN}$ or BLA for a total of $70 \mathrm{~min}$. After the initial $20 \mathrm{~min}$ of perfusion with either lidocaine or normal perfusate, the first meal of Fruit Loops was presented behind the screen. A second meal of the same food was presented 40 min after termination of lidocaine administration (i.e., when DA efflux had returned to baseline levels).

\section{Histology}

After the final microdialysis session, animals were anesthetized with chloral hydrate and perfused intracardially with $0.9 \%$ sodium chloride solution and then $3.7 \%$ paraformaldehyde solution. Brains were then removed and stored in Formalin with $20 \%$ w/v sucrose for a few days, sliced into $50 \mu \mathrm{m}$ coronal sections, and then stained with cresyl violet for verification of probe placements. Animals with tracts in both the (1) prelimbic-infralimbic region of the $\mathrm{mPFC}$ or shell-core region of the NAc and (2) CeN in the rostral amygdala or BLA in the caudal amygdala of both hemispheres were included in the statistical analyses.

\section{Data analyses}

Anticipatory behaviors. Anticipatory behaviors, defined as orienting and approach toward food placed behind a perforated screen, sniffing, and increased locomotor activity, were scored on a categorical rating of 1 , for display of anticipatory behavior, or 0 , for no behavior. These scores were then compared using the nonparametric statistic $\chi^{2}$.

Consummatory behavior and DA efflux. For the purposes of statistical analyses and graphical representation, DA efflux data were normalized to a baseline value (calculated by averaging the concentration of DA in the three samples preceding the final baseline sample). Consummatory behavior (i.e., amount of food consumed) and DA data were analyzed using repeated-measures ANOVAs, followed, when appropriate, by tests of simple main effects. In the latter tests, the significance level was adjusted according to the total number of tests to maintain a family-wise rate of type I error at $p<0.05$. DA data were further analyzed using Dunnett's method for comparisons from baseline and Dunn's tests for comparisons between the control and lidocaine conditions. The Huynh-Feldt correction for nonsphericity was applied to the degrees of freedom for all within-subject analyses. Statistical analyses were performed using Systat (Evanston, IL) or SPSS (Chicago, IL) statistical packages. 


\section{RESULTS}

\section{Basal concentration of DA in microdialysates collected from the MPFC and NAc}

The average concentration of DA uncorrected for probe recovery in the last three samples before administration of lidocaine by reverse dialysis in the $\mathrm{CeN}$ and the BLA were $0.14 \pm 0.19 \mathrm{~nm}$ in the mPFC (CeN-mPFC group) and $3.03 \pm 0.92 \mathrm{~nm}$ in the NAc (CeN-NAc and BLA-NAc groups).

\section{Effects of inactivation of the $\mathrm{CeN}$ on feeding behavior and DA efflux}

\section{Feeding behavior}

Reverse dialysis of lidocaine into the $\mathrm{CeN}$ increased overall intake of Froot Loops when compared with the normal perfusate condition in each of the $\mathrm{CeN}-\mathrm{NAc}$ and $\mathrm{CeN}-\mathrm{mPFC}$ groups $\left(F_{(7,49)}=3.555, p=0.004\right.$ and $F_{(7,56)}=3.286, p=0.005$, respectively). Because there was no significant difference in food intake between the two lidocaine conditions or between the two normal perfusate conditions, the data were collapsed across the two $\mathrm{CeN}$ groups into one lidocaine and one normal perfusate condition for subsequent statistical analyses. There was a significant interaction of condition $\times$ time on the amount of food consumed per $10 \mathrm{~min}$ over the two $40 \mathrm{~min}$ meals $\left(F_{(7,112)}=4.616\right.$; $p<0.001)$.

In the control condition (Figs. $1 A, 2 A$, bar graphs), fooddeprived rats were continuously dialyzed with normal perfusate in the $\mathrm{CeN}$ while they were presented with two meals of a highly palatable food, Froot Loops. Presentation of the initial meal behind a perforated screen elicited anticipatory behaviors ranging from sniffing and orienting toward the food bin behind the screen, approach toward the screen, as well as rearing and pacing in front of the screen. When the screen was removed, these control rats quickly approached the food and started to eat. There was a gradual decrease in the amount of food intake over the initial meal, a pattern consistent with the development of satiety. During the recess, when the screen was placed back into the testing chamber, animals were observed to return to a corner, groom, and then sleep. When a second meal of the same food was presented, animals did not show anticipatory responses toward the food source and, after removal of the screen, took much longer to approach the food bin. Occasional sampling of small amounts of food indicated the rats' satiated state. Overall, control rats consumed significantly more food during the first meal compared with the second meal (6.63 and 1.34 gm, respectively; $p<0.05$ ).

Inactivation of the $\mathrm{CeN}$ altered both anticipatory and consummatory behaviors associated with food. During administration of lidocaine into the $\mathrm{CeN}$, rats became aroused briefly and started to explore the testing chamber indiscriminately. In contrast to the control condition, none of the lidocaine-treated rats showed anticipatory responses toward the food $\left(\chi^{2}=34.0 ; p<0.001\right)$. Specifically, when the first meal of Froot Loops was presented behind the screen, rats did not make normal orienting or approach responses toward the food. In fact, several animals returned to a corner of the chamber and assumed a sleep posture or sat motionless.

When the screen was removed, lidocaine-treated rats took slightly longer than control rats to approach the food bin ( $>1$ vs $<1 \mathrm{~min}$, respectively) and consume the food. These rats manipulated and tasted each morsel of the food but they did not eat efficiently and repetitively discarded partially eaten pieces of Froot Loops. Furthermore, the rats did not show a typical decrease in the amount of intake over time associated with the

\section{A. CeN Perfusate/NAc DA}
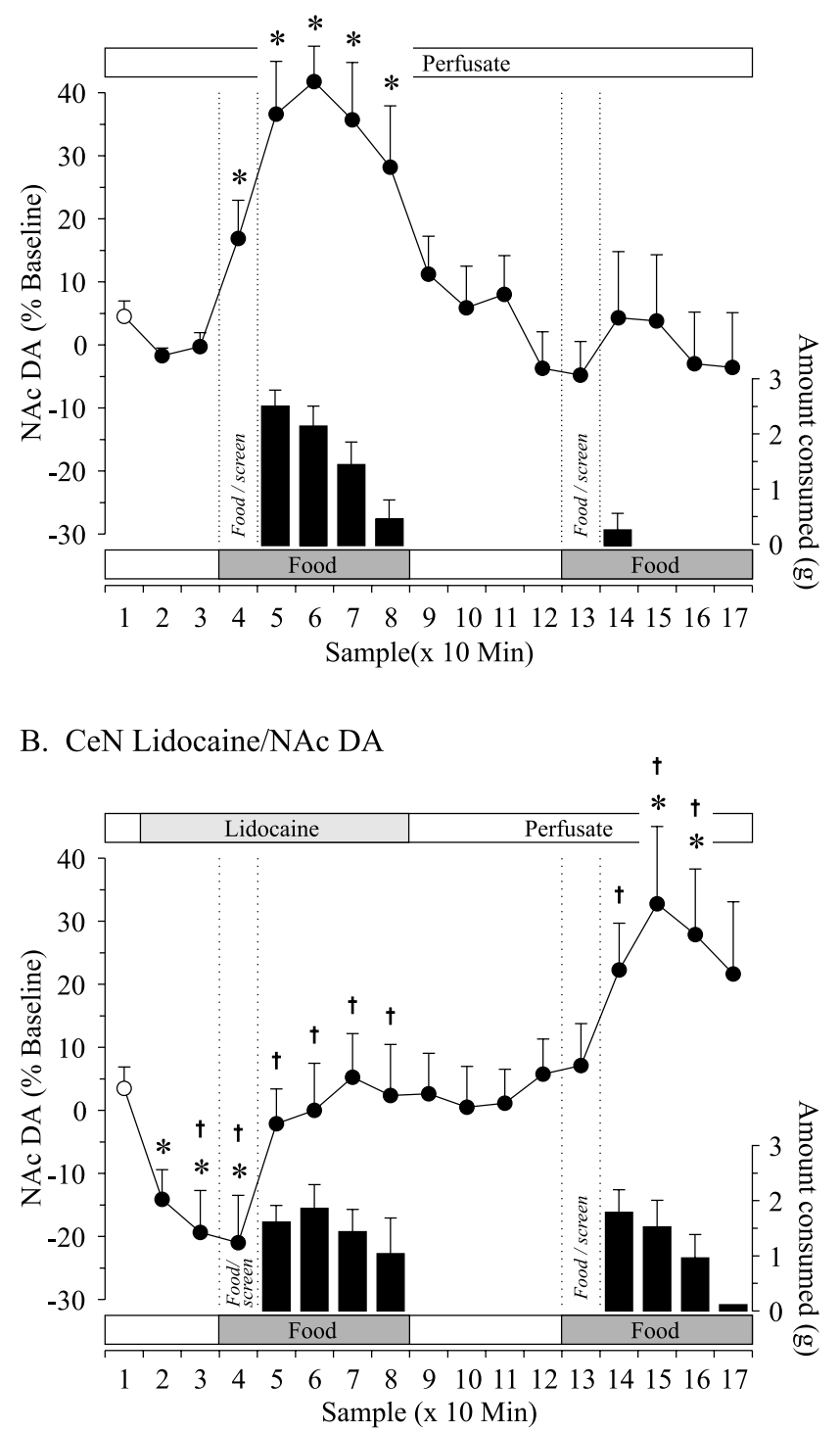

Figure 1. Effect of reversible inactivation of the CeN on DA efflux in the NAc associated with a food-devaluation protocol. Perfusion of the $\mathrm{CeN}$ with normal perfusate $(A ; n=8)$ or perfusate containing $2 \%$ lidocaine $(B ; n=8)$. Changes in DA efflux (line graph) and amount of food consumed (bar graph) per 10 min bins are represented as mean + SEM. Samples 4 and 13 represent periods during which a palatable food was presented behind a perforated screen, and changes in DA efflux during these periods are highlighted by dashed lines. Samples 5-8 and 14-17 represent periods during which animals had access to the food. Sample 1 (white circle) represents the baseline value used as the control mean in Dunnett's tests $\left({ }^{*} p<0.05\right)$. Comparisons between perfusate only and lidocaine conditions were conducted using Dunn's tests $(\dagger p<0.05)$.

development of satiety during a large extended meal (as reported by Ahn and Phillips, 1999). Rather, the amount consumed per 10 min remained relatively stable throughout the first meal (Figs. $1 B, 2 B$, bar graphs). After a $40 \mathrm{~min}$ recess, during which normal perfusate replaced the lidocaine in the probe, animals were presented with a second meal of Froot Loops behind the screen. This elicited normal anticipatory behaviors, including increased locomotion, approach toward the screen, and sniffing toward the food. After removal of the screen, all rats immediately approached the food bin and began to eat. A gradual decrease in amount of food intake during the second meal was consistent with 


\section{A. CeN Perfusate/mPFC DA}

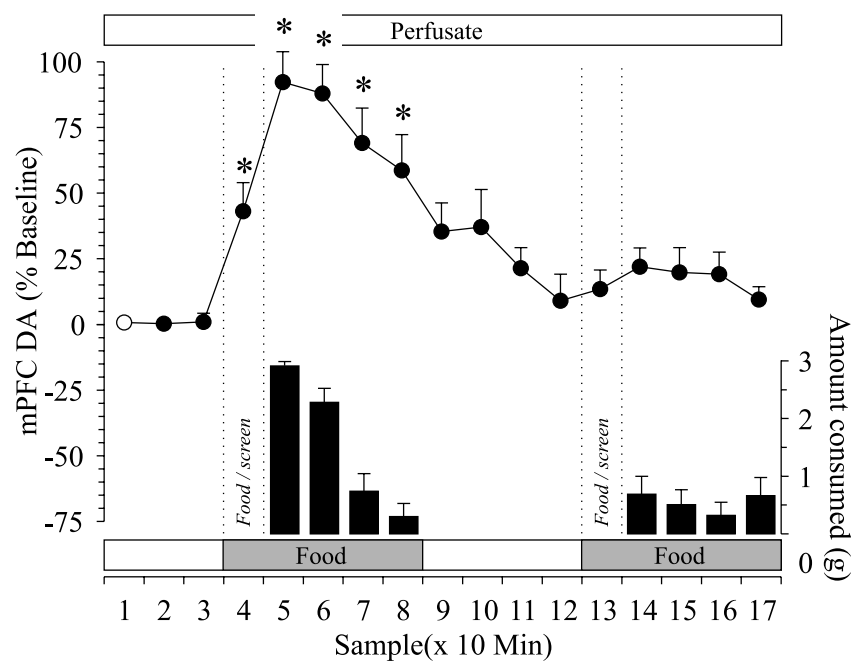

B. CeN Lidocaine/mPFC DA

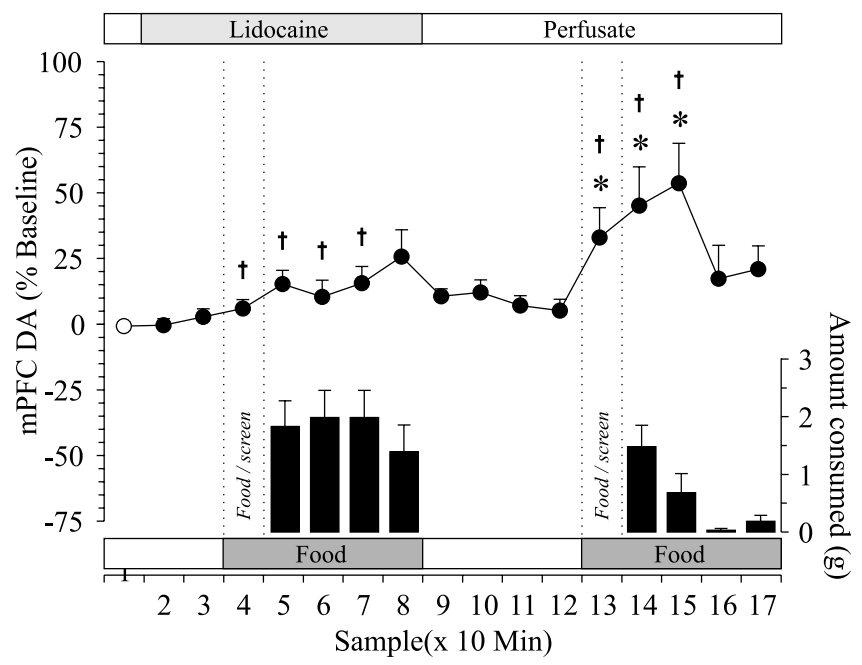

Figure 2. Effect of reversible inactivation of the CeN on DA efflux in the $\mathrm{mPFC}$ associated with a food-devaluation protocol. Perfusion of the $\mathrm{CeN}$ with normal perfusate $(A ; n=8)$ or $2 \%$ lidocaine $(B ; n=10)$. For additional explanation, see Figure 1 legend. ${ }^{*} p<0.05 ;{ }^{\dagger} p<0.05$.

the development of satiety. Although these lidocaine-treated rats consumed a significantly larger quantity of food during the initial meal (6.64 gm) compared with the second meal (3.34 gm; $p<$ $0.05)$, the amount consumed in the second meal was significantly greater than that consumed in the second meal by control subjects (1.34 gm; $p<0.05)$.

\section{$D A$ in the $N A c$}

A two-way repeated-measures ANOVA confirmed a significant condition $\times$ time interaction on DA efflux in the $\operatorname{NAc}\left(F_{(16,112)}=\right.$ 7.602; $p<0.001)$. Additional analyses revealed a simple main effect of time in both the lidocaine $\left(F_{(16,112)}=6.089 ; p<0.001\right)$ and control $\left(F_{(16,112)}=6.607 ; p<0.001\right)$ conditions.

Reverse dialysis of normal perfusate did not alter basal levels of DA efflux in the NAc (Fig. 1A, line graph). Presentation of the first meal behind the screen (the anticipatory period) was accom- panied by significant increases in DA efflux $(+17 \%$; $p<0.05)$. When the screen was removed and animals were allowed to consume the food (the consummatory period), there was an immediate significant increase in DA efflux that reached a maximal value of $+42 \%(p<0.05)$. This was followed by a gradual decline in DA efflux that mirrored the decreasing amount of food intake, and, during the recess period, DA levels returned to baseline values. Presentation of the second meal behind the screen or sampling of the food once the screen was removed failed to elicit additional changes in DA levels.

When lidocaine was reverse dialyzed into the $\mathrm{CeN}$, basal DA efflux in the NAc was decreased significantly to values $19-21 \%$ below baseline $(p<0.05)$ (Fig. $1 B$, line graph). While the $\mathrm{CeN}$ remained inactivated, presentation of the first meal behind a screen did not evoke any anticipatory changes in DA efflux (sample 4). Consumption of the first meal elicited a significant, albeit small, increase in DA efflux (+19\% from the suppressed basal DA levels; $p<0.05$ ) that remained at pre-lidocaine baseline levels through the first meal. At the start of the recess, replacement of lidocaine with normal perfusate in the $\mathrm{CeN}$ probes did not change DA efflux from baseline. Presentation of the same food as a second meal did not elicit anticipatory changes in DA efflux (sample 13); however, after consumption of this meal, there was a significant increase in DA efflux that reached a maximal value of $+33 \%(p<0.05)$. The subsequent decrease in amount of food intake per 10 min was accompanied by a gradual decline toward baseline in level of DA efflux.

\section{$D A$ in the $m P F C$}

A two-way repeated-measures ANOVA confirmed a significant condition $\times$ time interaction on DA efflux in the $\operatorname{mPFC}\left(F_{(16,128)}\right.$ $=8.331 ; p<0.001)$. Additional analysis revealed a simple main effect of time in both the lidocaine $\left(F_{(16,128)}=4.561 ; p<0.001\right)$ and control $\left(F_{(16,128)}=11.708 ; p<0.001\right)$ conditions.

In the control condition, administration of normal perfusate in the CeN did not alter basal levels of DA efflux in the mPFC (Fig. $2 A$, line graph). In general, the pattern of DA efflux in the $\mathrm{mPFC}$ closely mirrored the pattern of food intake. Presentation of the first meal elicited significant increases in DA efflux during the anticipatory period $(+43 \%$; $p<0.05)$. Removal of the screen was accompanied by a significant feeding-related increase in DA efflux $(+92 \% ; p<0.05)$ that gradually decreased toward baseline values as animals reached satiety for the food. During the second meal, neither anticipatory nor consummatory-related changes in DA efflux were observed.

In contrast to the effects on DA efflux in the NAc, administration of lidocaine into the $\mathrm{CeN}$ did not alter basal DA efflux in the mPFC (Fig. 2B, line graph) but did prevent anticipatory increases in DA efflux during the first meal (sample 4). Furthermore, as described above, the unusual pattern of food intake displayed by rats treated with lidocaine in the $\mathrm{CeN}$ was associated with an increase in DA efflux that was significantly attenuated compared with the control condition (+20 vs $+94 \%$ during the first $10 \mathrm{~min}$, respectively; $p<0.05)$. DA levels remained near baseline for the remainder of the meal, as well as throughout the recess period. Presentation of the second meal elicited significant increases in DA efflux associated with anticipatory behaviors $(+43 \% ; p<0.05)$ and consumption of food (maximal increase of $+58 \% ; p<0.05)$. 


\section{A. BLA Perfusate/NAc DA}

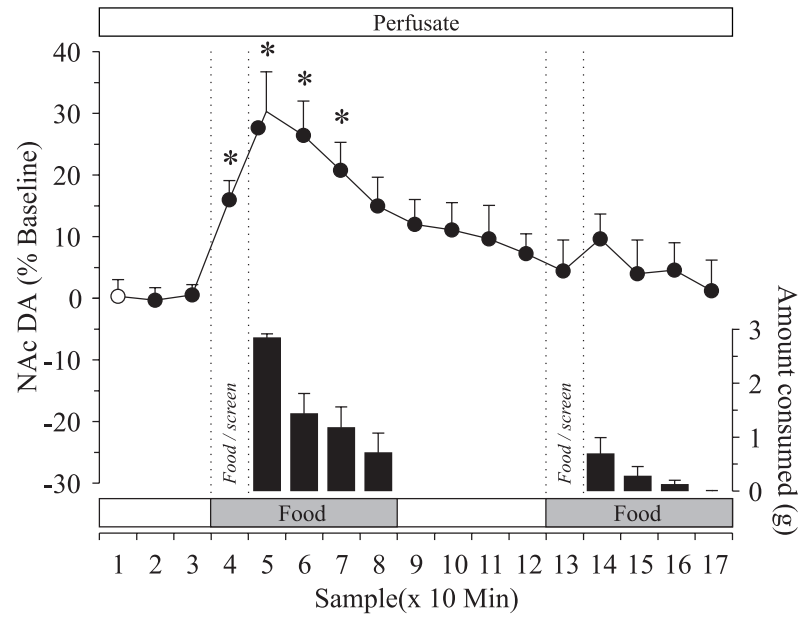

B. BLA Lidocaine/NAc DA

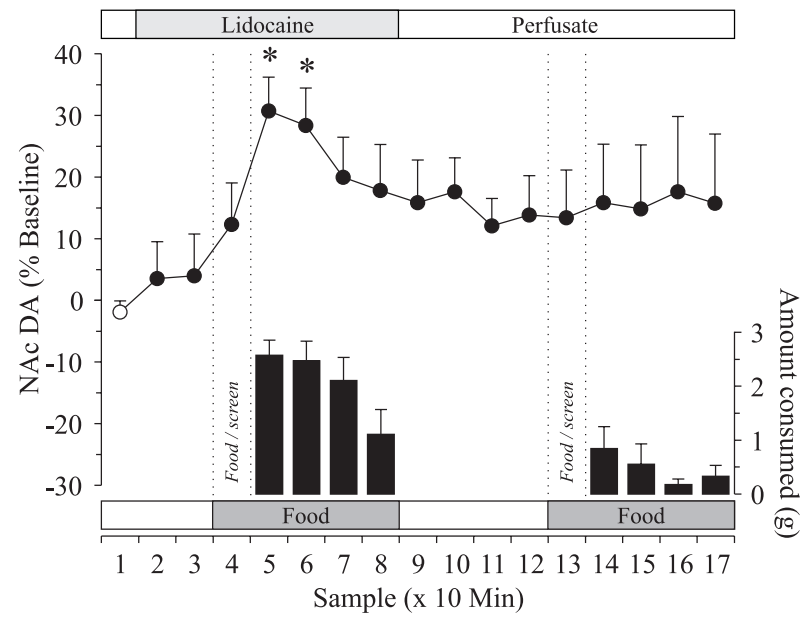

Figure 3. Effect of reversible inactivation of the BLA on DA efflux in the NAc during a food-devaluation protocol. Perfusion of the BLA with normal perfusate $(A ; n=8)$ or $2 \%$ lidocaine $(B ; n=9)$. For additional explanation, see Figure 1 legend. ${ }^{*} p<0.05$.

\section{Effects of inactivation of the BLA on feeding behavior and correlated DA efflux}

Feeding behavior

In contrast to the $\mathrm{CeN}$-lidocaine group, rats with BLA inactivation did not show behavioral activation coincident with lidocaine administration. No statistical differences in the pattern of food intake were observed between the lidocaine and control conditions $\left(F_{(1,8)}=2.918 ; p<0.126\right)$. Nevertheless, the slightly higher rate of consumption in the lidocaine compared with the control condition (Fig. 3, bar graphs) suggests that inactivation of the BLA may have some residual effect on satiety. Alternatively, these results may be viewed to fall within the normal range of variability in the pattern and amount of food intake, especially during the second and third bins, of the initial meal of other animals in the present study (Figs. $1 A, 2 A$ ). In this respect, a normal pattern of feeding to satiety was observed in both conditions, with rats consuming significantly less food during the second meal in the lidocaine $(8.3 \pm 1.3$ vs $1.9 \pm 0.9 \mathrm{gm} ; p<0.05)$ and control (6.1 \pm 1.1 vs $1.1 \pm 0.4$ gm; $p<0.05)$ conditions (Fig. 3, bar graphs).

\section{$D A$ in the $N A c$}

There were no statistically significant differences in DA efflux in the NAc between the lidocaine and control conditions $\left(F_{(1,8)}=\right.$ $0.848 ; p<0.384)$. There was a significant main effect of time in each of the lidocaine $\left(F_{(16,128)}=1.745 ; p<0.05\right)$ and control $\left(F_{(16,128)}=5.729 ; p<0.001\right)$ conditions. Presentation of the first meal behind the screen (Fig. 3, line graphs) elicited anticipatory increases in DA efflux that were significant in the control condition $(+16 \% ; p<0.05)$ but not in the lidocaine condition $(+12 \%)$. Consumption of the meal was associated with significant increases in DA efflux, with a maximal increase of +30 and $+31 \%$ in the lidocaine and control conditions, respectively $(p<0.05)$. The second presentation of the same meal did not elicit significant changes in DA efflux associated with anticipatory or consummatory phases of the experiment in either condition.

\section{Histology}

As illustrated in Figure 4, tracts left by microdialysis probes were located in the prelimbic-infralimbic region of the $\mathrm{mPFC}(+3.2$ to $+2.7 \mathrm{~mm} \mathrm{AP}$ ) or straddled the shell-core region of the NAc $(+2.2$ to $+1.6 \mathrm{~mm} \mathrm{AP})$. Tracts left by reverse-dialysis probes were located mainly in the $\mathrm{CeN}$ and but included some surrounding regions, such as the intercalated masses and substantia inominata $(-1.8$ to $-2.6 \mathrm{~mm} \mathrm{AP})$. BLA tracts were seen in the basal and lateral nuclei and, in two animals, in the lateral aspect of the caudal $\mathrm{CeN}(-2.8$ to $-3.3 \mathrm{~mm} \mathrm{AP})$.

\section{DISCUSSION}

\section{Separate mechanisms underlie the modulation of DA efflux by the CeN and BLA}

DA transmission in the mesocorticolimbic system is influenced by discrete manipulations of the $\mathrm{CeN}$ or the BLA (Floresco et al., 1998; Jackson and Moghaddam, 2001; Ahn and Phillips, 2002; Howland et al., 2002). The present effects on DA activity after lidocaine-induced inactivation of the $\mathrm{CeN}$ show that this region of the rat amygdala is involved in maintaining basal levels of DA efflux in the NAc and modulating feeding-evoked increases in DA efflux in both the NAc and mPFC. The same manipulation of the BLA, however, had little effect on basal or feeding-related changes in DA efflux in the NAc. In a previous study, we observed that inactivation of the BLA triggered large fluctuations in DA efflux in the mPFC, which indicates that the BLA may exert a stabilizing influence on basal levels of DA in the mPFC (Ahn and Phillips, 2002). A recent study from our laboratory used electrical stimulation to examine amygdala-DA interactions and reported a dissociation effect complementary to the present data (Howland et al., 2002). A brief $10 \mathrm{sec}$ stimulation of the BLA, but not the $\mathrm{CeN}$, caused a significant increase in DA efflux in the NAc. The combined results of these experiments suggest a double dissociation in the modulation of DA neurotransmission by the $\mathrm{CeN}$ and BLA. Specifically, basal or tonic levels of DA efflux are determined in part by tonic $\mathrm{CeN}$ inputs, whereas evoked or phasic increases in DA efflux are mainly influenced by excitatory inputs from the BLA.

The present results indicate that the $\mathrm{CeN}$ and the BLA belong to separate circuits that function in parallel to modulate the mesocorticolimbic DA system. The anatomical basis of this differential influence on DA efflux in the NAc and the mPFC most likely involves amygdalar projections to different levels of the DA 


\section{A. CeN/NAc group}
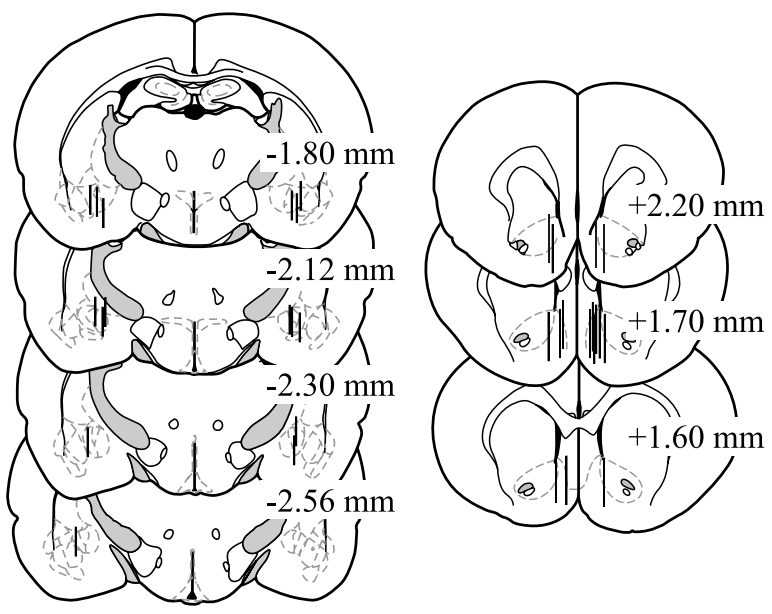

B. $\mathrm{CeN} / \mathrm{mPFC}$ group
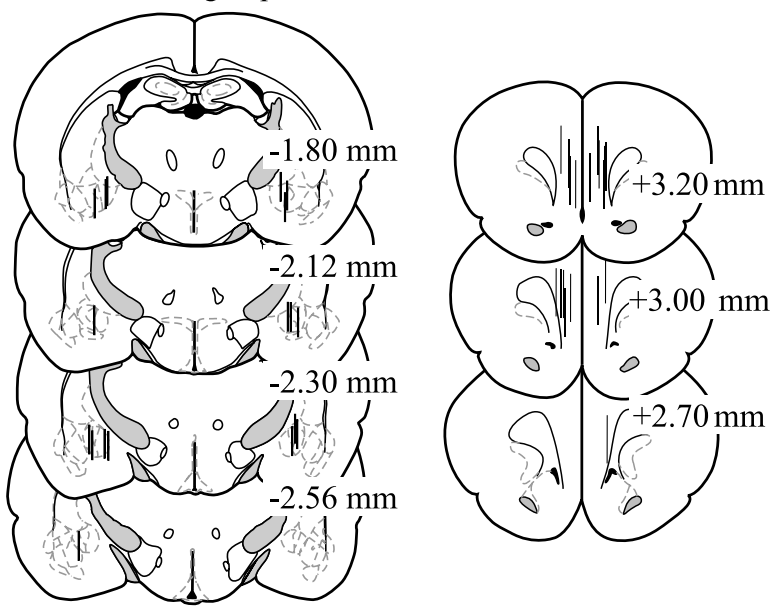

C. BLA/NAc group

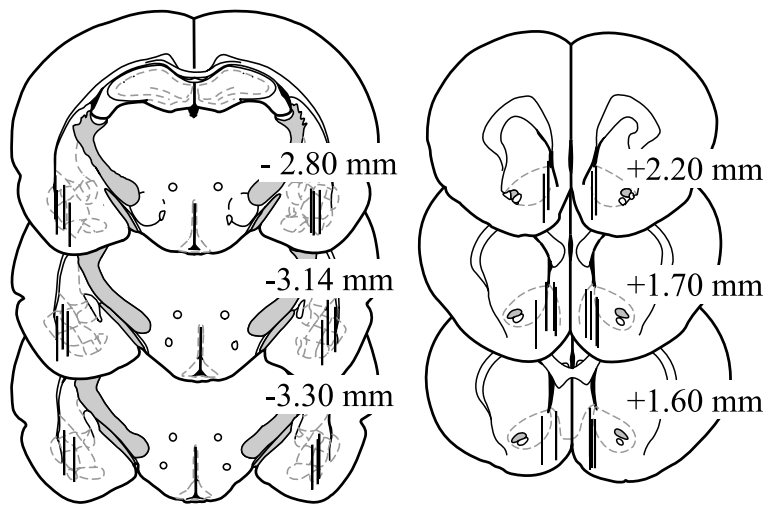

Figure 4. Location of reverse dialysis and microdialysis probes in the $\mathrm{CeN}$ and NAc $(A), \mathrm{CeN}$ and $\operatorname{mPFC}(B)$, and BLA and NAc $(C)$, respectively. Vertical lines represent the dialyzing lengths of microdialysis probes $(2.0 \mathrm{~mm})$ in the NAc and $\mathrm{mPFC}$ and reverse dialysis probes in the $\mathrm{CeN}(1.2 \mathrm{~mm})$ and BLA $(1.8 \mathrm{~mm})$. Drawings of coronal sections were adapted from Paxinos and Watson (1997). Distance from bregma is indicated.

system. Modulation of tonic levels of DA efflux in the NAc may involve a GABAergic projection from the $\mathrm{CeN}$ to the midbrain, including the ventral tegmental area, the substantia nigra, and the retrorubral field (Phillipson, 1979; Wallace et al., 1992; Sun and
Cassell, 1993). On the other hand, phasic changes in DA efflux may require excitatory inputs from the BLA, which have been shown to terminate in close proximity to DA varicosities in the NAc (Johnson et al., 1994). Thus, there exists in the NAc an anatomical arrangement consistent with a presynaptic mechanism for modulation of DA efflux by the BLA. A similar arrangement of BLA and DA inputs to the $\mathrm{mPFC}$ has yet to be demonstrated.

\section{Different roles for the CeN and BLA in food-related behaviors}

Although many behavioral responses are sensitive to manipulations of both the $\mathrm{CeN}$ and the BLA, performance of specific classes of behavior can be impaired by lesions of the $\mathrm{CeN}$ but not the BLA and vice versa (for review, see Cardinal et al., 2002). The present study used a free-feeding protocol that allowed rats to eat a palatable food to satiety during an initial meal and, during a subsequent meal, tested whether the reward value of the same food had been devalued by satiety. This protocol requires rats to make simple behavioral responses to the smell or taste of food, a process that may recruit pavlovian orienting or approach responses toward cues associated with food (Gallagher et al., 1990; Parkinson et al., 2000a). The present disruptive effects of inactivation of the $\mathrm{CeN}$ on anticipatory and consummatory measures and associated changes in DA efflux in the NAc are consistent with previous reports that the $\mathrm{CeN}-\mathrm{NAc}$ circuitry is involved in mediating approach responses toward incentive stimuli (Everitt et al., 1999). Furthermore, the attenuating effect of CeN inactivation on feeding-evoked DA efflux in the mPFC serves, according to our knowledge, as the first indication that the $\mathrm{CeN}$ and its influence on DA mechanisms in the mPFC may be a neural substrate of food reward.

In contrast to the $\mathrm{CeN}$, the BLA and its input to the NAc become critical in complex situations that involve learning voluntary instrumental actions to obtain both conditioned or natural rewards (Killcross et al., 1997; Everitt et al., 1999; Holland and Gallagher, 1999). For example, instrumental responding for food reward, second-order pavlovian conditioning, and conditioned reinforcement are all impaired after bilateral lesions of the BLA or functional dissociation of the BLA from the NAc via asymmetrical lesions (Simmons and Neill, 2001; Parkinson et al., 2000a; Setlow et al., 2002). The present data also provide indirect support for this proposed function, because inactivation of the BLA did not affect free feeding or feeding-evoked increases in DA efflux in the NAc. In general, the $\mathrm{CeN}$ appears to mediate stimulus-reward associations as occurs during feeding, whereas the BLA is involved when the value of reward must be used in conjunction with a learning rule to perform a task.

\section{Role of the CeN and its interaction with the DA system in food reward}

In the rat, the $\mathrm{CeN}$ receives important inputs involved in the regulation of food intake, including gustatory and olfactory information, as well as feedback regarding the motivational state of the animal (Mei, 1994; Zeigler, 1994; Norgren, 1995; Shipley et al., 1995; Woods et al., 1998). Accordingly, manipulations of the $\mathrm{CeN}$ have a wide range of effects related to feeding (Box and Mogenson, 1975; Galaverna et al., 1993; Hatfield et al., 1996). In the present study, inactivation of the $\mathrm{CeN}$ had two distinct effects. During the initial meal, rats failed to display a gradual decrease in food intake. During the second meal, when normal perfusate replaced lidocaine in the $\mathrm{CeN}$ and normal function was restored, rats surprisingly consumed a second meal of the same food 
presumably devalued to some degree, at least by preexposure, if not by satiety (Figs. $1 B, 2 B$, bar graphs). These observations suggest that temporary loss of $\mathrm{CeN}$ function impaired the development of satiety during an initial meal (a "resistance to satiety" effect) and, consequently, diminished the effect of devaluationby-satiety on intake of the same food.

Resistance to satiety may reflect a disruption of neural processes by which development of a satiety state devalues the hedonic qualities of the smell or taste of food. Alternatively, resistance to satiety may be attributable to a failure to establish an association between the sensory properties of a palatable food and the positive hedonic effect of consuming that food. It is noteworthy that, in monkeys, amygdala neurons that respond to food also respond to novel stimuli (Rolls, 1999). These electrophysiological data support Rolls' suggestion that "when relatively novel stimuli are encountered, they are investigated, e.g., by being smelled and then placed in the mouth, to assess whether the new stimuli are foods." In the absence of CeN function, animals may engage in excessive and prolonged oral examination of food, as observed in the present study and by Box and Mogenson (1975).

A key finding in the present study is that the $\mathrm{CeN}$ modulates important neurochemical correlates of free feeding, namely DA efflux in mesocorticolimbic terminal regions (Bassareo and Di Chiara, 1997; Ahn and Phillips, 1999). In the present study, inactivation of the $\mathrm{CeN}$ altered both anticipatory- and consummatory-related changes in DA efflux in the NAc and mPFC. Given the proposal that DA activity is closely linked to the incentive valence of food (Bassareo and Di Chiara, 1997; Berridge and Robinson, 1998; Ahn and Phillips, 1999), interaction between the $\mathrm{CeN}$ and the mesocorticolimbic DA activity may be a mechanism by which an animal's current state of hunger or satiety influences the incentive sensory properties of food (e.g., the olfactory or gustatory properties of food). Furthermore, the resistance to satiety effect after inactivation of the $\mathrm{CeN}$ suggests that this region of the rat amygdala mediates adjustments in behavioral responses to changes in incentive value of food (Galaverna et al., 1993; Salinas et al., 1996). However, this proposed role of the $\mathrm{CeN}$ remains controversial, because reinforcer devaluation effects have been reported to be unaffected by lesions of the CeN (Hatfield et al., 1996). In this regard, it is important that consummatory-related increases in DA efflux in the NAc and mPFC were attenuated rather than blocked during the second meal after reverse dialysis of lidocaine in the $\mathrm{CeN}$ (Figs. $1 B, 2 B$, line graphs). This raises the possibility that modulatory influences on DA activity associated with food reward may originate from other limbic regions, including the hippocampus and prefrontal cortex (Blaha et al., 1997; Parkinson et al., 2000b; Taepavarapruk et al., 2000).

The disruption of satiety in rats after inactivation of the $\mathrm{CeN}$ may also be related to the concept of "oral metering," which refers to the memory of what and how much has been eaten. This construct is exemplified in rare case studies, in which the eating behavior of densely amnesic but otherwise normal patients, including patient H.M., was carefully monitored (Hebben et al., 1985; Rozin et al., 1998). All had suffered damage to the mediotemporal lobe, including the hippocampus and amygdala. These individuals would consume a fairly large meal and, 10-20 min later, were unable to recall having the meal and, consequently, willingly accepted and consumed a second and sometimes a third identical meal (Rozin et al., 1998). In one instance, a subject reported "his stomach felt a little tight" before refusing a subsequent meal, suggesting that, within the physiological limits of satiety (i.e., physical fullness), memory for what has been consumed recently is an important determinant of the initiation and termination of food intake. Final acceptance of the hypothesis that the amygdala is involved in memory-based mediation of satiety must await evidence of similar effects in patients with selective damage to the amygdala.

\section{REFERENCES}

Aggleton JP, Passingham RE (1982) An assessment of the reinforcing properties of foods after amygdaloid lesions in rhesus monkeys. J Comp Phyiol Psychol 96:71-77.

Ahn S, Phillips AG (1999) Dopaminergic correlates of sensory-specific satiety in the medial prefrontal cortex and the nucleus accumbens of the rat. J Neurosci 19:RC29(1-6).

Ahn S, Phillips AG (2002) Independent modulation of basal and feeding-evoked dopamine efflux in the nucleus accumbens and medial prefrontal cortex by the central and basolateral amygdalar nuclei in the rat. Neuroscience, in press.

Bakshi VP, Kelley AE (1994) Sensitization and conditioning of feeding following multiple morphine microinjections into the nucleus accumbens. Brain Res 648:342-346.

Bassareo V, Di Chiara G (1997) Differential influence of associative and nonassociative learning mechanisms on the responsiveness of prefrontal and accumbal dopamine transmission to food stimuli in rats fed $a d$ libitum. J Neurosci 17:851-861.

Bechara A, van der Kooy D (1992) A single brain stem substrate mediates the motivational effects of both opiates and food in nondeprived rats but not in deprived rats. Behav Neurosci 106:351-363.

Berridge KC (1996) Food reward: brain substrates of wanting and liking. Neurosci Biobehav Rev 20:1-25.

Berridge KC, Robinson TE (1998) What is the role of dopamine in reward: hedonic impact, reward learning, or incentive salience? Brain Res Rev 28:309-369.

Berridge KC, Treit D (1986) Chlordiazepoxide directly enhances positive ingestive reactions in rats. Pharmacol Biochem Behav 24:217-221.

Blaha CD, Yang CR, Floresco SB, Barr AM, Phillips AG (1997) Stimulation of the ventral subiculum of the hippocampus evokes glutamate receptor-mediated changes in dopamine efflux in the rat nucleus accumbens. Eur J Neurosci 9:902-911.

Box BM, Mogenson GJ (1975) Alternations in ingestive behaviors after bilateral lesions of the amygdala in the rat. Physiol Behav 15:679-688.

Cardinal RN, Parkinson JA, Hall J, Everitt BJ (2002) Emotion and motivation: the role of the amygdala, ventral striatum, and prefrontal cortex. Neurosci Biobehav Rev 26:321-352.

Everitt BJ, Parkinson JA, Olmstead MC, Arroyo M, Robledo P, Robbins TW (1999) Associative processes in addiction and reward: the role of amygdala-ventral striatal subsystems. Ann NY Acad Sci 877:412-438.

Fiorino DF, Coury A, Phillips AG (1997) Dynamic changes in nucleus accumbens dopamine efflux during the Coolidge effect in male rats. J Neurosci 17:4849-4855.

Floresco SB, Yang CR, Phillips AG, Blaha CD (1998) Basolateral amygdala stimulation evokes glutamate receptor-dependent dopamine efflux in the nucleus accumbens of the anaesthetized rat. Eur $\mathbf{J}$ Neurosci 10:1241-1251.

Galaverna OG, Seeley RJ, Berridge KC, Grill HJ, Epstein AN, Schulkin J (1993) Lesions of the central nucleus of the amygdala. I. Effects on taste reactivity, taste aversion learning and sodium appetite. Behav Brain Res 59:11-17.

Gallagher M, Graham PW, Holland PC (1990) The amygdala central nucleus and appetitive pavlovian conditioning: lesions impair one class of conditioned behavior. J Neurosci 10:1906-1911.

Hatfield T, Han JS, Conley M, Gallagher M, Holland P (1996) Neurotoxic lesions of basolateral, but not central, amygdala interfere with pavlovian second-order conditioning and reinforcer devaluation effects. J Neurosci 16:5256-5265.

Hebben N, Corkin S, Eichenbaum H, Shedlack K (1985) Diminished ability to interpret and report internal states after bilateral medial temporal resection: case H. M. Behav Neurosci 99:1031-1039.

Holland PC, Gallagher M (1999) Amygdala circuitry in attentional and representational processes. Trends Cogn Sci 3:65-73.

Howland JG, Taepavarapruk P, Phillips AG (2002) Glutamate receptordependent modulation of dopamine efflux in the nucleus accumbens by basolateral, but not central, nucleus of the amygdala in rats. J Neurosci 22:1137-1145.

Jackson ME, Moghaddam B (2001) Amygdala regulation of nucleus accumbens dopamine output is governed by the prefrontal cortex. J Neurosci 21:676-681.

Johnson LR, Aylward RLM, Hussain Z, Totterdell S (1994) Input from the amygdala to the rat nucleus accumbens: its relationship with tyrosine hydroxylase immunoreactivity and identified neurons. Neuroscience 61:851-865. 
Kesner RP, Walser RD, Winzenried G (1989) Central but not basolateral amygdala mediates memory for positive affective experiences. Behav Brain Res 33:189-195.

Killcross S, Robbins TW, Everitt BJ (1997) Different types of fearconditioned behaviour mediated by separate nuclei within amygdala. Nature 388:377-380.

Maldonado-Irizarry CS, Swanson CJ, Kelley AE (1995) Glutamate receptors in the nucleus accumbens shell control feeding behavior via the lateral hypothalamus. J Neurosci 15:6779-6788.

Màlkovà L, Gaffan D, Murray EA (1997) Excitotoxic lesions of the amygdala fail to produce impairment in visual learning for auditory secondary reinforcement but interfere with reinforcer devaluation effects in rhesus monkeys. J Neurosci 17:6011-6020.

McDonald AJ (1991) Organization of amygdaloid projections to the prefrontal cortex and associated striatum in the rat. Neuroscience 44:1-14.

Mei N (1994) Role of digestive afferents in food intake regulation. In: Appetite: neural and behavioural bases (Legg CR, Booth D, eds), pp 86-97. Oxford: Oxford UP.

Mogenson GJ, Phillips AG (1976) Motivation: a psychological construct in search of a physiological substrate. Prog Psychobiol Physiol Psychol $6: 189-243$

Murray EA, Gaffan EA, Flint Jr RW (1996) Anterior rhinal cortex and amygdala: dissociation of their contributions to memory and food preference in rhesus monkeys. Behav Neurosci 110:30-42.

Norgren R (1995) Gustatory system. In: The rat nervous system (Paxinos G, ed), pp 751-771. San Diego: Academic.

Parkinson JA, Robbins TW, Everitt BJ (2000a) Dissociable roles of the central and basolateral amygdala in appetitive emotional learning. Eur J Neurosci 12:405-413.

Parkinson JA, Cardinal RN, Everitt BJ (2000b) Limbic cortical-ventral striatal systems underlying appetitive conditioning. Prog Brain Res 126:263-285.

Paxinos G, Watson C (1997) The rat brain in stereotaxic coordinates. Toronto: Academic.

Phillipson OT (1979) Afferent projections to the ventral tegmental area of Tsai and interfascicular nucleus: a horseradish peroxidase study in the rat. J Comp Neurol 187:117-144.

Pitkänen A, Savander V, LeDoux JE (1997) Organization of intraamygdaloid circuitries in the rat: an emerging framework for understanding functions of the amygdala. Trends Neurosci 20:517-523.
Robinson TE, Berridge KC (1993) The neural basis of drug craving: an incentive-sensitization theory of addiction. Brain Res Rev 18:247-291.

Rolls ET (1999) The brain control of feeding and reward. In: The brain and emotion, pp 8-58. New York: Oxford UP.

Rozin P, Dow S, Moscovitch M, Rajaram S (1998) What causes human beings to begin and end a meal? A role for memory for what has been eaten, as evidenced by a study of multiple meal eating in amnesic patients. Psychol Sci 9:392-396.

Salinas JA, Parent MB, McGaugh JL (1996) Ibotenic acid lesions of the amygdala basolateral complex or central nucleus differentially effect the response to reductions in reward. Brain Res 742:283-293.

Setlow B, Holland PC, Gallagher M (2002) Disconnection of the basolateral amygdala complex and nucleus accumbens impairs appetitive pavlovian second-order conditioned responses. Behav Neurosci 116:267-275.

Shipley MT, McLean JH, Ennis M (1995) Olfactory system. In: The rat nervous system (Paxinos G, ed), pp 899-926. San Diego: Academic.

Simmons DA, Neill DB (2001) Functional interaction between the basolateral amygdala and the ventral striatum underlies incentive motivation of a food reward. Soc Neurosci Abstr 26:959.15.

Stratford TR, Kelley AE (1997) GABA in the nucleus accumbens shell participates in the central regulation of feeding behavior. J Neurosci 17:4434-4440.

Sun N, Cassell MD (1993) Intrinsic GABAergic neurons in the rat central extended amygdala. J Comp Neurol 330:381-404.

Swanson LW, Petrovich GD (1998) What is the amygdala? Trends Neurosci 21:323-331.

Taepavarapruk P, Floresco SB, Phillips AG (2000) Hyperlocomotion and increased dopamine efflux in the rat nucleus accumbens evoked by electrical stimulation of the ventral subiculum: role of ionotropic glutamate and dopamine $\mathrm{D}_{1}$ receptors. Psychopharmacology 151:242-251.

Uwano T, Nishijo H, Ono T, Tamura R (1995) Neuronal responsiveness to various sensory stimuli, and associative learning in the rat amygdala. Neuroscience 68:339-361.

Wallace DM, Magnuson DJ, Gray TS (1992) Organization of amygdaloid projections to brainstem dopaminergic, noradrenergic, and adrenergic cell groups in the rat. Brain Res Bull 28:447-454.

Woods SC, Seeley RJ, Porte Jr D, Schwartz MW (1998) Signals that regulate food intake and energy homeostasis. Science 280:1378-1383.

Zeigler HP (1994) Brainstem orosensorimotor mechanisms and the neural control of ingestive behaviour. In: Appetite: neural and behavioural bases (Legg CR, Booth D, eds), pp 54-85. Oxford: Oxford UP. 